\section{Acute epiglottitis in adults}

Sir,

Acute epiglottitis, usually caused by Haemophilus influenzae type B occurs only rarely in adults. ${ }^{1}$ We have treated 15 such patients ( $18-60$ years; $8<35$ years) over a period of 6 years (1986-1991) in Medical College \& Hospital, Rohtak, India.

All were males. Ten presented within 48 hours of onset of symptoms while five had had symptoms for 3-4 days before reporting. Pain or difficulty in swallowing was the main symptom (14 cases). Pain in the throat (six cases), respiratory distress (five cases) and change in voice (four cases) were other presenting symptoms. All were febrile and had a red swollen epiglottis. Inflammation of aryepiglottic folds and arytenoids was observed in seven cases and tonsillopharyngitis in six. In five cases vocal cords could not be visualized. The important differential diagnosis included tubercular epiglottitis and malignancy, excluded by history and relevant examination. X-ray soft tissue neck showed the swollen epiglottis producing a 'thumb sign' in 14 patients. In 10 cases where throat swab and blood culture was done, the blood culture was sterile in all, while throat swab showed Klebsiella pneumoniae (sensitive to ampicillin, gentamicin and kanamicin) in three. All patients improved with parenteral ampicillin. The mean hospital stay was 5.2 days (3-10 days).

As in the present study, acute epiglottitis has been reported to have a greater preponderance among males. ${ }^{2}$ The incidence of the 'thumb sign' seen in the present study is also similar to that reported earlier. ${ }^{3}$ Other radiological findings reported include prevertebral swelling with obliteration of the vallecular space and ballooning of the hypopharynx. These were, however, not seen in present series.

It has been said that acute epiglottitis is associated with septicaemia. The isolation of an organism from both blood and epiglottis is a better indicator of its pathogenic role than cultures from epiglottis alone, where many incidental throat commensals are to be found.$^{4}$ In the present study $K$. pneumoniae was isolated in three cases only. The possibility of a viral aetiology in the rest cannot be ruled out.

In addition to antibiotics, proper airway management is the mainstay of treatment. Endotracheal intubation should be done when obstruction is imminent. ${ }^{4}$ Acute epiglottitis runs a much more benign clinical course in adults as compared to children ${ }^{5}$ and unlike children no respiratory distress occurs due to examination. None of our patients required tracheostomy/endotracheal intubation and no complication or recurrence was seen over a follow-up period of 3 months.

Ishwar Singh' S.P.S. Yadav U. Wig

S. Sarkar Department of Otolaryngology. Medical College \& Hospital, Rohtak and ' $802 / 22$, Opp. NFL Area Office,

Jhang Colony,

Rohtak, India.

\section{References}

1. Maran, A.G.D. Laryngeal stridor. In: Maran, A.G.D. (ed.) Logan Turner's Disease of the Nose, Throat and Ear. Varghese Company, Bombay, 1988, pp. 381-391.

2. Morgenstein, K.M. \& Abramson, A.L. Acute epiglottitis in adults. Laryngoscope 1971, 81: 1066-1073.

3. Stanley, R.E. \& Liang, T.S. Acute epiglottitis in adults (The Singapore experience). J Laryngol Otol 1988, 102: 1017-1021.

4. Turk, D.C. Acute epiglottitis in an adult (Letter). Br Med J 1976, 2: 1385.

5. Arndal, H. \& Andrassen, U.K. Acute epiglottitis in children and adults. Nasotracheal intubation, tracheostomy or careful observation? Current status in Scandinavia. J Laryngol Otol 1988, 102: 1012-1016.

\section{Pharyngeal pouch presenting as dysphagia after a stroke}

Sir,

We report a patient who developed dysphagia following an acute stroke, in whom a diagnosis of a previously asymptomatic pharyngeal pouch was made and in whom normal swallowing returned when the pouch was resected.

An 83 year old previously fit man developed sudden onset of weakness and speech disturbance. On examination he was drowsy, dysphasic, dysarthric with an absent gag reflex and he had an incomplete right hemiparesis. A stroke was diagnosed, food and oral fluids were withheld and he was hydrated intravenously. On the third day he had sitting balance and was continent of urine, but a speech therapist felt that he was still at risk of aspiration. By day 8 his hemiparesis had improved and he was mobile with the aid of one person, but considerable dysarthria and dysphasia persisted and when tested he was still unable to swallow safely. On the 10th day a fine bore nasogastric tube was passed (on the first attempt) and feeding was commenced. On the 24th day the nasogastric tube had become dislodged and it was not possible to pass it satisfactorily again because it became coiled in the oesophagus at each attempt. By this time he had become independently mobile on the flat with a stick, his dysarthria and dysphasia had improved, but when his swallowing was tested he appeared to aspirate then regurgitate. It was decided to perform a percutaneous endoscopic gastrostomy on the 26th day. However, the endoscope could not be passed beyond a large pharyngeal pouch, the extent of which was subsequently confirmed by a barium swallow. Even at this stage no history of any swallowing disturbance before the stroke could be obtained. He was operated upon 7 days later when the pouch was approached externally and a diverticulectomy and cricopharyngeal myotomy was performed without problems. By 6 days postoperatively he was eating and drinking normally and was fully independent in the activities of daily living.

Dysphagia due to a pharyngeal pouch is not common but, like stroke, predominantly affects the elderly. ${ }^{1}$ In our patient it remains a matter of speculation whether the stroke merely unmasked a pre-existing but previously asymptomatic pouch, of permitted a small, asymptomatic, pouch to enlarge sufficiently to become symptomatic. Percutaneous endoscopic gastrostomy is a safe and effective procedure ${ }^{2}$ and would have been our initial 
treatment of choice, but eventual surgical treatment of the pharyngeal pouch would have been recommended because of the continuing risk of aspiration and pouch enlargement.

Most cases of dysphagia after hemispheric stroke resolve within two weeks of onset. ${ }^{3}$ However, physicians should be aware of non-neurogenic causes of dysphagia in their patients with stroke and should investigate dysphagia when it is out of proportion to the other neurological deficits.

\section{J.R.F. Gladman R.M. Kupfer P.J. Bradley Department of Health Care of the Elderly, University Hospital, Nottingham NG7 $2 U H, U K$.}

\section{References}

1. Bowdler, D.A. Pharyngeal pouches. In: Kerr, A.G. (ed.) Scott-Brown's Otolaryngology, 5th ed. Butterworth, London, 1987, pp. 264-282.

2. Park, R.H.R., Allison, M.C., Lang, J. et al. Randomised comparison of percutaneous endoscopic gastrostomy and nasogastric tube feeding in patients with persisting neurological dysphagia. Br Med J 1992, 304: 1406-1409.

3. Gordon, C., Langton Hewer, R. \& Wade, D.T. Dysphagia in acute stroke. $\mathrm{Br}$ Med J 1987, 2,5: 411-414.

\section{Spontaneous haemorrhage into a pituitary tumour after streptokinase therapy}

Sir,

A 47 year old bank clerk awoke one morning with severe chest pain radiating to both arms and was found to have suffered an acute anterior non-transmural myocardial infarction. He was treated with 1.5 million units of streptokinase intravenously over 1 hour together with 5,000 units of calcium heparin subcutaneously and $300 \mathrm{mg}$ aspirin by mouth. Two hours after finishing the streptokinase infusion, he complained of sudden severe headache, diplopia and blurred vision. On examination, he was fully conscious with right third and sixth nerve palsies, left temporal scotoma and bilateral limited upward gaze. Thrombin time: 29 seconds (normal range 7-12); fibrinogen concentration: $0.5 \mathrm{~g} / \mathrm{l}$ (normal range $2-4 \mathrm{~g} / \mathrm{l}$ ); prothrombin time: 19 seconds (normal range 10-14); partial thromboplastin time: 47 seconds (normal range $35-45$ ).

An urgent cranial computed tomographic (CT) scan revealed a large enhancing pituitary mass with suprasellar extension. A fluid level, with high density posteriorly, was present within the central portion of the tumour indicating recent haemorrhage. Serum prolactin: $213,000 \mathrm{mU} / 1$ rising to $304,500 \mathrm{mU} / 1$ after thyrotrophin releasing hormone stimulation; basal and stimulated serum cortisol and thyrotrophin were normal.
Bromocriptine (1.25 mg daily) was commenced and over the following 3 days his headache disappeared. The cranial nerve palsies recovered after one month. Six months later, he was well and his prolactin level was now $84 \mathrm{mU} / \mathrm{l}$. The previously demonstrated fluid levels were no longer visible on CT scan and the pituitary tumour was much reduced in size.

Bleeding complications in the large trials of streptokinase in acute myocardial infarction were infrequent but patient selection was strict with only $14-35 \%$ of patients eligible for treatment., ${ }^{1,2}$ As the use of thrombolytic treatment becomes more widespread, the incidence of haemorrhagic complications may be expected to rise. Haemorrhage may be more likely into a vascular tumour and has been reported in association with a renal carcinoma. $^{3}$

Bleeding into a pituitary tumour has not been previously described after streptokinase but has been reported after anticoagulant treatment. ${ }^{4}$ Early diagnosis is essential because of the high mortality of untreated cases. $^{5}$ Reversal of antithrombotic therapy has been recommended when bleeding problems arise but could aggravate the underlying thrombotic episode for which they were prescribed. When pituitary apoplexy arises, we urge rapid referral to a suitable centre where full evaluation and monitoring can be performed.

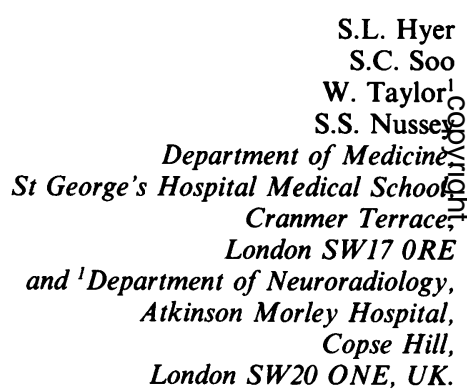

\section{References}

1. Gruppo Italiano per lo Studio della Streptokinasi Nell'infarto Miocardico (GISSI). Effectiveness of intravenous thrombolytic treatment in acute myocardial infarction. Lancet 1986, i: 397-402.

2. ISIS-2 (Second International Study of Infarct Survival) Collaborative Group. Randomised trial of intravenous streptokinase, oral aspirin, both, or neither among 17,187 cases of suspected acute myocardial infarction: ISIS-2. Lancet 1988, ii: $349-360$.

3. Johnston, R.T., Jameson, J.S. \& Macpherson, D.S. Retroperitoneal haemorrhage from a renal carcinoma in association with streptokinase therapy. Postgrad Med J 1991, 67: 10291030 .

4. Nourizadeh, A.R. \& Pitts, F.W. Haemorrhage into pituitary adenoma during anticoagulant therapy. JAMA 1965, 193: 623-625

5. Cardoso, E. \& Peterson, E. Pituitary apoplexy: a review. Neurosurgery 1984, 14: 363-373. 